Title:

\title{
Choosing diverse sets of plausible scenarios in multidimensional exploratory futures techniques
}

Authors:

\author{
S. Lord, A. Helfgott, J. Vervoort
}

Environmental Change Institute, University of Oxford, Oxford, UK

\begin{abstract}
:
Morphological analysis allows any number of dimensions to be retained when framing future conditions, and techniques within morphological analysis determine which combinations of those dimensions represent plausible futures. However, even a relatively low number of dimensions in future conditions can lead to hundreds or even thousands of plausible future scenarios. Creating highly diverse but conceivable visions of the future in which to explore decision-making, exploratory futures techniques rely on the selection of a small number of plausible scenarios from the larger set. In this paper we describe a new method for finding maximally diverse sets containing a small number of plausible scenarios from a multi-dimensional morphological analysis. It is based on a mathematical optimization of diversity that is robust to the uncertainty in the framing of future factors and states and in what stakeholders might consider diverse combinations of those factors and states. We also describe implementation of the method as a software tool and its performance in recent exploratory scenario development by CGIAR and partners for regional environmental change, food security and livelihoods.
\end{abstract}

Keywords:

morphological analysis; scenario diversity; exploratory scenarios; regional environmental change scenarios; robust optimization; OLDFAR

Highlights:

- new method to extract a maximally diverse set of scenarios from morphological analysis

- use of robust optimization to account for uncertainty in diversity between scenarios

- users provided with multiple sets of diverse scenarios for qualitative assessment

- implemented in graphic user interface software tool for use in participatory workshops

- key lessons learned through application in four regional scenario case studies with CGIAR and 240 partner organizations 
Body:

\section{Introduction}

Complexity and uncertainty are dominant features of many public and private decision-making issues [1]. The success of any decision in these situations is more often than not shaped by diverse actors, with differing perspectives and agendas, at multiple levels within changing interrelated social, economic, political and environmental systems [2, 3]. Exploratory scenarios are particularly effective tools for incorporating high degrees of complexity and uncertainty into planning and decision-making processes [4-10].

This paper introduces a new exploratory scenario method called OLDFAR used in participatory scenario development processes conducted by the CGIAR program on Climate Change, Agriculture and Food Security (CCAFS'1). The CGIAR program has been developing socioscenarios for East Africa, West Africa, South Asia, Southeast Asia, the Andes and Central America together with regional experts from 240 organizations in all sectors related to rural livelihoods, environments, climate adaptation and mitigation, agricultural development and food security. These scenarios have been used with decision-makers at regional (or sub-global) and national levels to help test the feasibility of policies and investments and guide priority setting, largely related to government policy but also with non-governmental organizations, development banks and private sector investors. The reference [4] describes the overall scenarios process and main lessons in terms of effective engagement with decision-makers. This paper provides the mathematical details of the OLDFAR method and discusses its application, benefits and drawbacks, both theoretical and as experienced in practice in the CGIAR program. A separate paper will discuss the broader considerations and theory around the design of scenarios in the process used in the CGIAR regional scenarios program.

Morphological Analysis [11-16], in particular, Field Anomaly Relaxation (FAR) was chosen for the CGIAR program. FAR considers, in the case of the CGIAR program through participatory discussion, the important dimensions of future conditions (hereafter called factors) and mutually exclusive instantiations of each factor (hereafter called states of each factor). Incompatible combinations of states, also called implausible in the literature, are ruled out, leaving all remaining combinations of states as plausible future scenarios. Previous participatory regional environmental change, food security and livelihoods scenarios studies have mostly used the "two axis" or "quadrant" approach of two factors with two extreme states each, which provide four scenarios [17-19]. While this scenario development method has the benefits of being simple to conduct and understand, it runs the risk of strongly limiting and framing views of the future by these two factors and two states - leading to a limited exploration of the future possibility space and, in multi-stakeholder contexts, to perceptions of limited usability of the scenarios when potential users see key factors as under-represented. FAR is a multi-dimensional exploratory scenario technique which places no conceptual limit on the numbers of factors or states within factors.

\footnotetext{
${ }^{1}$ http://ccafs.cgiar.org/
} 
The choice of factors and states in the FAR method are transparent. Alternative multidimensional techniques, such as the so-called "inductive" method which has been used by Royal Dutch Shell [20] and others, where scenarios are generated in an emergent, organic fashion, building on combining narrative elements by "trend crashing" and identifying relevant scenario narratives first, and only then providing an overall structure to the scenario set, has the drawback that the choices about which factors and states are explored, and which are ignored, are often intransparent.

In the context of interacting, multi-dimensional socio-economic and environmental changes, assigning specific probabilities to long-term scenarios that cover large geographic/political areas is problematic $[1,21]$. That FAR is based on plausibility, not probability, was another reason for its adoption by the CGIAR program. Plausibility emphasizes the need for scenarios to be believable from the subjective perspectives of scenario developers and users [22]. Ensuring that scenarios are plausible to users is important to help them expand their perspectives on the future [6] - if scenarios are considered to be too implausible, users will respond by narrowing, rather than broadening, the future possibility space they are willing to consider. Exploratory scenarios derived from the FAR method therefore may be different from, for instance, extreme scenarios that they might consider extremely unlikely, though not impossible, future scenarios.

To create highly diverse but conceivable visions of the future in which to explore decisionmaking, exploratory futures techniques rely on the selection of a small number of plausible scenarios from the larger set with highly distinct narratives and back-casting. Having highly diverse and distinct scenarios is important because scenarios are often used to test the robustness of strategies, for priority-setting and to develop contingency plans. With these purposes in mind, and working from a notion that complexity and uncertainty make the assignment of exact probabilities difficult and often dangerous, more distinct and diverse scenarios allow for a more comprehensive assessment of the viability of strategies [19]. When more factors and states are taken into consideration when structuring scenarios, the possibility space of the scenarios set increases. However, there are practical limitations to how many factors and states can be taken into account.

Applying the method described in this paper in South Asia we found that participants found six factors hard to remember when it came to quantification of the scenarios and we decided to use less factors in subsequent processes. Preliminary evidence suggests that participants in multifactor, multi-state processes are more comfortable with a lower number of factors but a higher number of potential states. This way, participants have to consider less factor interactions, but a higher number of states per factor increases the scenario set's possibility space. In addition, with fewer factors and more states, not all states will end up in the final scenario set, reducing the complexity of the set.

Even a low-dimensional morphological analysis can contain hundreds and potentially thousands of plausible scenarios. Practice shows that between four and six scenarios is the best number for qualitative participatory exploration of highly uncertain futures, since this places the number of factors under the cognitive load rule of seven [23] beyond which individuals find it hard to keep distinct mental objects in mind together. Too few scenarios will not provide a sufficiently diverse set, and may additionally lead to participants viewing one scenario as the "good" scenario the other as the "bad" scenario, creating a one-dimensional scenario set. A set of 3 scenarios tends to support participants' consideration of one scenario as the "likely" scenario, 
the "baseline", et cetera. With too many scenarios that each represent multi-dimensional narratives on the future, scenario developers and users will lose sight of the whole and lose their sense of how the scenarios are distinct from one another, making the set a less accessible tool for decision guidance [10].

Generally, participants agreed with the above rationale for choosing four to six highly diverse scenarios from a larger set of thousands as the basis for developing further an exploratory scenario set. However, this does not imply that participants agreed on what makes scenarios diverse and how diverse one scenario is with respect to another. The notion of diversity is itself subjective, and with participants from many organizations and sectors the CGIAR program thought it had additional value to incorporate a wider view of what it meant to be highly diverse. Just as multidimensional analysis was used to allow more scope in the "important" factors, and so generate more agreement and acceptance from participants that the exploratory scenarios did cover changes in important future aspects, it was desirable to also allow multiple representations of diversity between scenarios so that there would be agreement and acceptance from participants that the scenarios were indeed diverse, and, ideally, that from each of their viewpoints they were highly diverse.

The basic problem therefore is how to choose four to six highly diverse scenarios from a larger set of thousands where there is uncertainty in the notion of diversity. If there was a fixed notion of diversity (no uncertainty) then mathematical optimization is a valuable tool for the first part of the problem. As an illustration, an early scenario analysis conducted by the authors in South Asia [4] involved six future factors, with four, four, three, four, four, and six states within the factors respectively. From the 4608 scenarios in the total morphological space, the FAR method determined 1322 plausible scenarios. There are over 7.3 quadrillion $\left(7.3 \times 10^{15}\right)$ choices of 6 scenarios from the 1322 plausible scenarios. There are still over 1.3 billion $\left(1.3 \times 10^{9}\right)$ choices of 4 scenarios from the 422 most plausible scenarios in the top 25 percent of FAR scores. Heuristic examination can reduce the number of combinations by orders of magnitude and argue that certain selections of scenarios are sufficiently diverse for exploration. However, within a multidimensional futures space with large numbers of combinations it is difficult for heuristic methods to demonstrably satisfy the exploratory requirement for highly diverse and distinct scenarios. A highly diverse set, to be identified as such, requires comparison with a maximally diverse set of scenarios which attempts to minimize the aspects of the multi-dimensional future not being explored. Except in the simplest cases, a heuristic argument cannot guarantee comparison with maximal diversity while that maximal diversity sits un-estimated within millions of combinations.

Add an additional heuristic examination for agreement on diversity into the above process and it becomes difficult and complex. This is why we developed the OLDFAR method. There are mathematical tools for finding so-called robust solutions in optimization problems, where the objectives to be optimized themselves have uncertainty [58-66]. These robust optimization techniques can be applied to try and find a small subset of scenarios that would be highly diverse according to multiple views of diversity.

\section{OLDFAR method}

We introduce a robust optimization technique on top of the FAR method called Optimized Linear Diversity Field Anomaly Relaxation (OLDFAR) to produce highly diverse sets of future 
scenarios for exploratory futures analysis. For an example, consider the set of factors and states from a participatory process in Table 1 . The CGIAR method for identifying and distilling uncertain and import future factors, and reinserting rich content from participant's discussions after a diverse set of scenarios is chosen, is reported elsewhere, [4,76,77],

Table 1: An example FAR array of future factors (columns) and potential states (rows)

\begin{tabular}{|l|l|l|l|l|l|}
\hline $\begin{array}{l}\text { E: } \\
\text { Economic }\end{array}$ & $\begin{array}{l}\text { S: } \\
\text { Political } \\
\text { Stability }\end{array}$ & $\begin{array}{l}\text { P: } \\
\text { Projection of } \\
\text { Power }\end{array}$ & $\begin{array}{l}\text { A: } \\
\text { Regional Co- } \\
\text { operation }\end{array}$ & $\begin{array}{l}\text { R: } \\
\text { Resource } \\
\text { Pressures }\end{array}$ & $\begin{array}{l}\text { China's } \\
\text { Attitude }\end{array}$ \\
\hline $\begin{array}{l}\text { E1: rosy } \\
\text { growth }\end{array}$ & $\begin{array}{l}\text { S1: strong and } \\
\text { stable }\end{array}$ & $\begin{array}{l}\text { P1: effective } \\
\text { and very } \\
\text { influential }\end{array}$ & $\begin{array}{l}\text { A1: close co- } \\
\text { operation }\end{array}$ & $\begin{array}{l}\text { R1: low } \\
\text { pressure }\end{array}$ & $\begin{array}{l}\text { C1: leader and } \\
\text { policemand }\end{array}$ \\
\hline E2: fair growth & $\begin{array}{l}\text { S2: fairly } \\
\text { stable }\end{array}$ & $\begin{array}{l}\text { P2: fairly } \\
\text { influential }\end{array}$ & $\begin{array}{l}\text { A2: loose } \\
\text { multi- } \\
\text { lateralism }\end{array}$ & $\begin{array}{l}\text { R2: moderate } \\
\text { pressure }\end{array}$ & C2: one of us \\
\hline $\begin{array}{l}\text { E3: stifled } \\
\text { growth }\end{array}$ & S3: shaky & $\begin{array}{l}\text { P3: limited } \\
\text { influence }\end{array}$ & $\begin{array}{l}\text { A3: no multi- } \\
\text { lateralism }\end{array}$ & $\begin{array}{l}\text { R3: high } \\
\text { pressure }\end{array}$ & $\begin{array}{l}\text { C3: minds own } \\
\text { business }\end{array}$ \\
\hline $\begin{array}{l}\text { E4: negative } \\
\text { growth }\end{array}$ & S4: unstable & & A4: enmity & $\begin{array}{l}\text { R4: crisis } \\
\text { situation }\end{array}$ & $\begin{array}{l}\text { C4: pushy, } \\
\text { verbally }\end{array}$ \\
\hline & & & $\begin{array}{l}\text { C5: forceful, } \\
\text { militarily }\end{array}$ \\
\hline
\end{tabular}

The FAR method determines which strings of states, e.g. $(1,1,1,1,1,1)$ representing (E1,S1,P1,A1,R1,C1), or $(3,2,1,1,2,6)$ representing (E3,S2,P1,A1,R2,C6), are compatible and represent a plausible scenario for the six future factors. As mentioned, for this set of factors (as columns) and states (as rows) there are $7.3 \times 10^{15}$ different ways to pick six plausible scenarios. Which choices of six represent the most diverse futures? Diversity is a comparison between scenarios; one scenario is diverse only in regard to another. Quantitative comparison is formalized in mathematics by the notion of a metric [24]. Diversity within a set of scenarios is derived from the minimal inter-spacing between its elements. Uncertainty in: the framing of future factors and states; what stakeholders might consider diverse combinations of those factors and states; the elicitation process if diversity metrics are elicited from participants; or whether a generic diversity metric best reflects the participants views if diversity metrics are not elicited, means that we do not choose a single diversity metric. We assume a set of diversity metrics and a prior indexed by the same set, where the prior is a subjective belief about the uncertainties mentioned $[25,26]$. The OLDFAR method itself does not distinguish between how the set of metrics or prior is obtained, they may be based on generic principles or elicited directly from participants. Analysis for the CGIAR workshops used a generic set of metrics as described in Section 4. In most applications the prior will be a uniform distribution, representing, according to the principle of maximum entropy, complete uncertainty or lack of knowledge about which diversity metric is a better reflection [27, 28].

Sets containing the desired number of exploratory scenarios (in the example above, sets of six plausible scenarios) are measured against all diversity metrics simultaneously. Combining this collection of diversity measurements with the prior produces, for each such set of scenarios, a distribution that represents its diversity score rather than a single number [25, 26, 29]. That the diversity score for a set of scenarios is a distribution reflects the uncertainty in measuring diversity. To identify sets of plausible scenarios that are maximally diverse in this setting we use 
the idea of robust optimization. There are many robust optimization methods, but we use a simple robust optimization principle going back to Markowitz involving the expected value and variance of the distribution [67]. Examination of the Pareto front in multi-objective optimization [30-32] to maximize the expected diversity score and minimize the variance of the diversity score yields sets of scenarios with robust maximal diversity. These sets of scenarios are returned to stakeholders as candidate sets of exploratory future scenarios for evaluation.

Incorporating uncertainty in the measurement of diversity and performing a robust optimization of diversity provides a margin. Whichever of the metrics might turn out to best reflect the relation between factors, and the subjective view of stakeholders about what makes the scenarios diverse, the robust maximal solution will perform as relatively well, or as "highly" as possible. A robust solution may not be the optimal, that is, the "most diverse", for any particular stakeholder, [33-35]. Robust optimization has been used previously in futures analysis, particularly in the context of risk and future decisions, [36-40]. Similar principles to OLDFAR are used in alternative and complementary mathematical analytic methods for generating small sets of scenarios from a broadly represented future uncertainty [41-45]. References $[42,43]$ use a "scenario discovery" approach that seeks a small set of scenarios that best differentiate those futures in which proposed policies meet or do not meet specified goals. The selection of scenarios is immediately policy relevant, but contingent on the particular policies under consideration. Exploratory methods like OLDFAR are complementary for an initial exploration and generation of policy ideas. They also offer an alternative evaluation method of particular policies without the utility-like specification of goals or values required to program the scenario discovery. References $[41,45]$ use the same principle of optimization to maximize diversity posterior to morphological analysis to derive a smaller set of scenarios. OLDFAR considers uncertainty in the diversity (allowing a more general range of diversity metrics than in $[41,45]$ ) and is distinct as a method using robust optimization posterior to morphological analysis.

It is important to note that the mathematics in the OLDFAR technique is only for leverage on the number of choices of a small number of plausible scenarios from a larger set. As described and implemented it takes as input a human judgment based FAR analysis, and multiple candidate sets of the desired number of exploratory scenarios are provided back to the users so that human judgment makes the final decision on what is the most useful set of scenarios to go forward with for exploratory analysis. The leverage provided by OLDFAR has proven useful even when the number of plausible scenarios is not as large as the CCAFS South Asia study, as discussed briefly below and in more detail in a separate paper in the development of scenarios for regional environmental change, food security and livelihoods in South East Asia, the Andes and Central America.

In the following sections we describe the mathematical principles of OLDFAR, its implementation in a software tools for use in the CGIAR program, some example outputs, and the benefits and drawbacks learnt in practice.

\section{Mathematics of OLDFAR}

We describe the mathematical details of OLDFAR that produces candidate sets of exploratory scenarios from a multi-dimensional FAR morphological analysis. 


\subsection{Plausible future scenarios from Field Anomaly Relaxation}

From a morphological analysis let $N$ be the number of factors with $m_{1}, \ldots, m_{N}$ mutually exclusive states in the factors, respectively. The scenario space represented by

$$
S:=\mathbb{Z}_{m_{1}} \times \ldots \times \mathbb{Z}_{m_{N}}
$$

has $m_{1} \times \ldots \times m_{N}$ elements. The scoring

$$
f: S \rightarrow[0,1]
$$

from a field anomaly relaxation (FAR) is meant to assign to each scenario in the scenario space the confidence that the future states within the scenario can occur together. This is referred to variously as the compatibility of the states or the plausibility of the scenario. It is a measure of the confidence that the scenario could exist, [15], it is not a measure of the probability that the future, if that scenario could exist, will be that scenario. A $f$ score of 0 indicates that the participants think that the future states are not compatible, and a $f$ score of 1 indicates that the participants think that the future states are compatible ${ }^{2}$.

A FAR score $f: S \rightarrow[0,1]$ means that the scenarios in $S$ can be ordered by plausibility, i.e. $s>t$ if $f(s)>f(t)$, which is a total preorder [48]. Denote the set of plausible scenarios by

$$
P S:=\{s \in S: f(s)>0\}=\operatorname{supp} f .
$$

In a multi-dimensional morphological analysis, $P S$ may contain hundreds or thousands of scenarios. The total preorder order induced on $S$ by $f$ means that one can restrict to smaller sets of "more" plausible scenarios, for example, by looking only at the scenarios in the upper quartile of FAR scores. The upper quartile will generally still contain hundreds or thousands of scenarios. Further reduction to a smaller set of scenarios using plausibility becomes counterproductive. The significant ambiguity in the FAR scores means that one should not look just at a small set of maximum FAR scored scenarios and consider them to be the only plausible futures.

\subsection{Diversity score of a set of plausible scenarios}

Diversity is a comparison between scenarios of how different is the future they are exploring based purely on the partitioning of the future given by the morphological analysis. Mathematically, diversity is represented by a family of metrics on the set of scenarios $[24,49]$

$$
\rho(\theta, \because,): S \times S \rightarrow[0, \infty), \quad \theta \in \Theta .
$$

The indexing set $\Theta$ represents uncertainty in the framing of future factors and states and in what stakeholders might consider diverse combinations of those factors and states. A particular family of metrics that we have used in practice is described in Section 4. A prior $p$ on $\Theta$ represents subjective belief about the degree to which a diversity metric in the family reflects diversity as assessed by the stakeholders $[25,26,29,50]$. Mathematically the prior is a probability measure on $\Theta$ [51-54]. To avoid reference to measure theory in the following

\footnotetext{
${ }^{2}$ Technically, output from FAR should map a scenario to subjective confidence measured by a distribution on not compatible or compatible $[25,26]$, or belonging to not compatible or compatible $[46,47]$.
} 
formulas we assume that $\Theta$ is a discrete set so that all probability measures are associated to weights. In most applications the prior is specified by a uniform weighting [27, 28, 55].

Define the sets of $k$ plausible scenarios,

$$
P S_{k}:=\left\{\left\{s_{1}, \ldots, s_{k}\right\}: s_{i} \in P S, i=1, \ldots, k\right\} .
$$

Recall that elements in sets are unordered, so a permutation of the same scenarios is the same set of $k$ plausible scenarios. Define the diversity scoring functions $d(\cdot, \cdot): \Theta \times P S_{k} \rightarrow[0,1]$ by

$$
d(\theta, R):=\frac{\min _{s, t \in R, s \neq t} \rho(\theta, s, t)}{\max _{R^{\prime} \subset P S_{k}} \min _{s^{\prime}, t^{\prime} \in R^{\prime}, s^{\prime} \neq t^{\prime}} \rho\left(\theta, s^{\prime}, t^{\prime}\right)}, \quad \theta \in \Theta, R \in P S_{k} .
$$

Using the diversity scoring functions we can now push-forward the prior $p$ to a distribution of diversity scores on $[0,1]$, [53]. For example, if $p$ is a uniform weighting then distribution of diversity scores is a set of weightings on the range of $\Theta$ under $d(\cdot, R)$. For convenience of notation we turn the weightings into a formal distribution on $[0,1]$ using delta functions $[56$, 57]. That is, for a set $R$ of $k$ plausible scenarios define its distribution of diversity scores as $\backslash$

$$
\operatorname{div}(\lambda, R):=\sum_{\left\{\lambda^{\prime}: \exists \theta \in \Theta, d(\theta, R)=\lambda^{\prime}\right\}\left\{\theta \in \Theta: d(\theta, R)=\lambda^{\prime}\right\}} p(\theta) \delta\left(\lambda-\lambda^{\prime}\right), \quad \lambda \in[0,1] .
$$

We refer to the distribution $\operatorname{div}(\cdot, R)$ on $[0,1]$ as the diversity score of the set $R$ of $k$ plausible scenarios.

\subsection{Robust maximally diverse scenarios}

A combination of high expected value and low variance in the diversity score indicates that the set has high diversity scores and that this fact is generally insensitive to the uncertainty. Mathematically, we are seeking to find sets of $k$ plausible scenarios that maximize the objective function $E: P S_{k} \rightarrow[0,1]$,

$$
E(R):=\int_{0}^{1} \lambda \operatorname{div}(\lambda, R) d \lambda, \quad R \in P S_{k}
$$

and minimize the objective function $V: P S_{k} \rightarrow[0,1]$,

$$
V(R):=\int_{0}^{1}(\lambda-E(R))^{2} \operatorname{div}(\lambda, R) d \lambda, \quad R \in P S_{k}
$$

Our method of robust optimization is, in this way, reduced to a standard two-criterion optimization problem [30-32, 68].

Normally the two-criterion optimization would be performed on the sets of $k$ plausible scenarios whose diversity score is not stochastically dominated by some other set of $k$ plausible scenarios. In the current setting $\operatorname{div}(\cdot, R)$ stochastically dominates $\operatorname{div}\left(\cdot, R^{\prime}\right)$, written $R>R^{\prime}$, [69], if for all $\mu \in[0,1]$

$$
\int_{0}^{\mu} \operatorname{div}(\lambda, R)-\operatorname{div}\left(\lambda, R^{\prime}\right) d \lambda \leq 0, \quad R, R^{\prime} \in P S_{k}
$$


and strict inequality for some $\mu \in[0,1]$. If $R>R^{\prime}$ then irrespective of the uncertainty $R$ will always be believed to have higher diversity than $R^{\prime}$. Variance, as a criterion for robustness of high values to uncertainty, becomes a redundant objective to minimize on dominated sets (Figure 1). The non-dominated sets are where the expected value and variance are in relevant trade-off for robust maximization and the full Pareto front is of interest.

However, the quantitative diversity score is not the only assessment criteria on sets of $k$ plausible scenarios relevant. The morphological space has qualitative description and meaning for each factor and each state in a factor, and the combined narrative of a set of $k$ plausible scenarios is a qualitative criterion that also assesses the diversity of the set of scenarios for exploratory analysis. OLDFAR uses the quantitative score as initial leverage to reduce the potentially vast number of sets of $k$ plausible scenarios to a list where the qualitative assessment of narrative can be performed. Removing dominated sets removes the sets with evidently redundant diversity score (Figure 1c in comparison to Figure 1b), but may remove some "near" robust optimal sets of $k$ plausible scenarios whose combined narrative might be of high interest for further exploration.

\section{INSERT FIGURE 1}

\subsection{Selecting candidates for narrative assessment}

So that both diversity and combined narrative can be assessed, OLDFAR returns up to $n$ choices of robust "near" optimal sets of $k$ plausable scenarios with distinction between combined narratives. Approaches to do this depend somewhat on the way OLDFAR is implemented. We describe two approaches.

The first approach, which can be implemented by a software interface, is for human users to determine distinction in narrative and the criteria of robust "near" optimal. The software displays the diversity scores and the narratives of the Pareto optimal front [30,31] of robust highly diverse sets of $k$ plausible scenarios (evidently redundant sets can be removed from consideration by the program using a weakened stochastic dominance order or introducing a penalized version of the variance objective function). Human users compare the diversity scores for closeness to robust maximal diversity and then the narratives for distinction to already selected candidates. Large Pareto fronts are displayed in portions with members (or representative members from equal-space clusters along the front) selected at random without replacement. If the list of candidates has not reached $n$ after examining the Pareto front, the robust optimization is iterated with the previous front removed. The iteration stops when $n$ candidates are chosen or when the human users assess that the new front contains sets no longer close enough to robust maximal diversity. The candidate final set is appealing, since it represents our original intention of "high" diversity, which has been compared with the maximal diversity in a way that it is difficult for heuristic argument on its own to achieve.

The second approach is to assess distinction in narrative by the proxy of diversity between sets of $k$ plausible scenarios. Subsets that have a diversity of elements within them do not necessarily have much diversity between them. Consider the two sets $\{1,10,5,7\}$ and $\{1,10,5,8\}$ 
of 4 choices of numbers between and including 1 and 10. Each set is highly inter-spaced but compared to each other they are not very different. In the second approach OLDFAR robustly maximizes, amongst $n$ sets of $k$ plausible scenarios, the diversity between the $n$ sets as well as the diversity within each set of $k$ scenarios. This involves adding a dimension to theoptimization of the last section. We describe it briefly.

Define the $n$ sets of $k$ plausible scenarios,

$$
P S_{n, k}:=\left\{\left\{R_{1}, \ldots, R_{n}\right\}: R_{i} \in P S_{k}, i=1, \ldots, n\right\} .
$$

The within diversity score function of a $n$ set of $k$ scenarios uses the least diversity score function of $k$ scenarios,

$$
d_{w}(\theta, \mathcal{A}):=\min _{R \in \mathcal{A}} d(\theta, R), \quad \theta \in \Theta, \mathcal{A} \in P S_{n, k} .
$$

For the between diversity score define the family of metrics on $P S_{k}$ by

$$
\rho_{b}(\theta, Q, R):=\sum_{s \in Q} \min _{t \in R} \rho(\theta, s, t)+\sum_{t \in R} \min _{s \in Q} \rho(\theta, s, t), \quad \theta \in \Theta, R, S \in P S_{k}
$$

and the between diversity scoring functions of an $n$ set of $k$ scenarios by

$$
d_{b}(\theta, \mathcal{A}):=\frac{\min _{R, Q \in \mathcal{A}, s \neq t} \rho_{b}(\theta, R, Q)}{\max _{\mathcal{A}^{\prime} \subset P S_{n, k} R^{\prime}, Q^{\prime} \in \mathcal{A}^{\prime}, s^{\prime} \neq t^{\prime}} \rho_{b}\left(\theta, R^{\prime}, Q^{\prime}\right)}, \quad \theta \in \Theta, \mathcal{A} \in P S_{n, k} .
$$

Using the diversity scoring functions we can now push-forward the prior $p$ to a distribution of diversity scores on the square $[0,1]^{2}$. For $\lambda, \sigma \in[0,1]$ and $\mathcal{A} \in P S_{n, k}$ define

$$
\operatorname{div}(\lambda, \sigma, \mathcal{A}):=\sum_{\left\{\left(\lambda^{\prime}, \sigma^{\prime}\right): \exists \theta \in \Theta,\right.} \sum_{\left.\begin{array}{l}
d_{w}(\theta, \mathcal{A})=\lambda^{\prime} \\
d_{b}(\theta, \mathcal{A})=\sigma^{\prime}
\end{array}\right\}\left\{\begin{array}{l}
d_{w}(\theta, \mathcal{A})=\lambda^{\prime} \\
d_{b}(\theta, \mathcal{A})=\sigma^{\prime}
\end{array}\right\}} p(\theta) \delta\left(\lambda-\lambda^{\prime}\right) \delta\left(\sigma-\sigma^{\prime}\right) .
$$

The distribution $\operatorname{div}(\cdot,, \mathcal{A})$ on $[0,1]^{2}$ reflects the within and between diversity score of the $n$ set of $k$ scenarios with uncertainty.

We seek to maximize the objective functions $E_{w}, E_{b}: P S_{n, k} \rightarrow[0,1]$,

$$
\begin{array}{ll}
E_{w}(\mathcal{A}):=\int_{0}^{1} \lambda \operatorname{div}(\lambda, \sigma, \mathcal{A}) d \lambda d \sigma, & \mathcal{A} \in P S_{n, k} \\
E_{b}(\mathcal{A}):=\int_{0}^{1} \sigma \operatorname{div}(\lambda, \sigma, \mathcal{A}) d \lambda d \sigma, & \mathcal{A} \in P S_{n, k}
\end{array}
$$

and minimize the objective function $V: P S_{n, k} \rightarrow[0,1]$

$$
V(\mathcal{A}):=\frac{1}{2} \int_{0}^{1}\left(\left(\lambda-E_{w}(\mathcal{A})\right)^{2}+\left(\sigma-E_{b}(\mathcal{A})\right)^{2}\right) \operatorname{div}(\lambda, \sigma, \mathcal{A}) d \lambda, \quad \mathcal{A} \in P S_{n, k}
$$

Balanced solutions to this three-criterion optimization problem with removal of dominated sets (see below) provide a robust conclusion of high expected diversity within the $k$ scenario sets 
(scenarios that minimize the future not being explored) and high expected diversity between the $n$ sets of $k$ scenarios (distinct combined narratives between those scenario sets minimizing the future not being explored). In the software implementation we allowed users to control the preferential choice from the Pareto front. This is detailed in the Appendix. They could choose to favor a robust high within diversity, a robust high between diversity, or a balance across all three criteria.

As before for distributions on [0,1], appropriate trade-off between the three-criterion requires dominated distributions $\operatorname{div}(\cdot, \cdot, \mathcal{A})$ on $[0,1]^{2}$ to be removed. There are several versions of stochastic dominance that can be used for distributions on the square $[0,1]^{2},[69,70]$. Using the standard version of lower orthant order, we say $\operatorname{div}(\cdot,, \mathcal{A})$ stochastically dominates $\operatorname{div}\left(\cdot,,, \mathcal{A}^{\prime}\right)$, written $\mathcal{A}>_{2} \mathcal{A}^{\prime}$, if for all $\left(\mu_{1}, \mu_{2}\right) \in[0,1]^{2}$

$$
\int_{0}^{\mu_{1}} \int_{0}^{\mu_{2}} \operatorname{div}(\lambda, \sigma, \mathcal{A})-\operatorname{div}\left(\lambda, \sigma, \mathcal{A}^{\prime}\right) d \lambda d \sigma \leq 0, \quad \mathcal{A}, \mathcal{A}^{\prime} \in P S_{n, k}
$$

and strict inequality for some $\mu_{1}, \mu_{2} \in[0,1]$. The interpretation of dominance is that if $\mathcal{A}>_{2} \mathcal{A}^{\prime}$ then the $n$ set of $k$ scenarios $\mathcal{A}$ is always believed to have higher combined choices of diversity within and diversity between.

\subsection{Exploratory future scenarios from OLDFAR}

After choosing from the non-dominated $\mathcal{A} \in P S_{n, k}$ in the Pareto front OLDFAR outputs at this stage a $n$ set of $k$ plausable future scenarios. Each set of $k$ plausable future scenarios robustly minimizes the futures not being explored when extracting $k$ scenarios from the initial morphological space. The $n$ choices represent distinct combined narratives. Human judgment can now assess the diversity and combined narrative and decide which single set of $k$ plausable future scenarios to proceed with as exploratory scenarios.

\section{Implementation of OLDFAR}

For generating exploratory scenarios in CGIAR futures workshops, we implemented OLDFAR in a software graphic user interface. The interface consisted of three parts.

Firstly, users use an interface to enter FAR results in the form of the FAR factor compatibility matrices and a skeleton narrative (a shortened description) for each factor and each state of each factor. Users save this information.

The second user interface calculates number of plausible scenarios from the information entered and, provided there are greater than two plausible scenarios, allows users to choose OLDFAR analysis options. Choosing an initial scenario is not mandatory to the OLDFAR method, but it is a feature of participatory workshops and provides a computational advantage. The current OLDFAR software implements the second method of assessing narrative diversity, so the analysis options interface also asks users for preference for a $n$ set of $k$ scenarios that favors a robust high within diversity, a robust high between diversity, or a balance of high within and between diversity and robustness (the three preferences are specified further in Figure 2 below). Users can explore the output of all three preferences by returning from the analysis results interface back to the analysis option interface and repeating the analysis. 
Once the analysis is initiated by a user, a third interface displays the OLDFAR result of a $n$ set of $k$ scenarios with the within diversity score distributions and combined skeleton narratives.

Users can compare the diversity score and the combined narrative across the $n$ set to choose a single set of $k$ exploratory scenarios. Alternatively, the results can be saved and printed as text and pdf output for later examination.

The software program, also called OLDFAR, incorporating the above interfaces and necessary algorithms was written as a Matlab GUI ${ }^{3}$. OLDFAR currently has English and Spanish versions and operates as a standalone executable when packaged with the Matlab runtime library. Packages are available for download in Windows, Linux and Mac platforms. A web-based version is under construction. The program places limits on OLDFAR parameters, $N=6$ (maximum number of factors is 6), $m_{i}=10, i=1, \ldots, N$, (maximum number of states in each factor is 10 ), $k=9$ (maximum output number of exploratory scenarios), $n=4$ (the number of alternative sets of $k$ scenarios displayed).

\subsection{Diversity metrics used in software implementation}

The OLDFAR computer program implements the OLDFAR method using the following generic family of metrics for diversity.

Using the notation of the Section 3, define for $s, t \in S$

$$
\Delta(s, t)_{i}:=\frac{s_{i}-t_{i}}{m_{i}-1}, \quad i=1, \ldots, N
$$

and for $\Theta:=\{\alpha, \beta, \gamma>0: \alpha+\beta+\gamma=1\}$

$$
\rho((\alpha, \beta, \gamma), s, t):=\frac{\alpha}{N} \underbrace{\sum_{i=1}^{N}\left|\Delta(s, t)_{i}\right|}_{\begin{array}{c}
\text { magnitude change } \\
\text { in factors }
\end{array}}+\frac{\beta}{N} \underbrace{\sum_{i=1}^{N} \chi_{(0,1]}\left(\left|\Delta(s, t)_{i}\right|\right)}_{\begin{array}{c}
\text { magnitude change } \\
\text { across factors }
\end{array}}+\underbrace{\frac{\gamma}{N} \sum_{i=1}^{N}\left|\Delta(s, t)_{i}-\overline{\Delta(s, t)}\right|}_{\begin{array}{c}
\text { deviation change } \\
\text { in factors }
\end{array}}
$$

where $\overline{\Delta(s, t)}$ is the average of the vector $\Delta(s, t)$. The first term indicates the amount of state change in each of the factors, the second term indicates what factors have changed, and the third term indicates the deviation in the state change. The combination of first and second terms makes change across factors relatively more significant to diversity than change in factors, e.g. if $N=4, m_{1}=5=m_{2}=m_{3}=m_{4}$ then

$$
\rho((\alpha, \beta, \gamma),(1,1,1,1),(3,1,1,1))<\rho((\alpha, \beta, \gamma),(1,1,1,1),(2,2,1,1)), \quad \beta>0,
$$

and the third term makes a mixture of state change relatively more significant to diversity than a progression of state change, e.g.

$$
\rho((\alpha, \beta, \gamma),(1,2,3,4),(2,3,4,5))<\rho((\alpha, \beta, \gamma),(1,2,3,4),(2,1,4,3)), \quad \gamma>0 .
$$

\footnotetext{
${ }^{3}$ Windows Matlab R2014a, Mac Matlab R2012b, Linux Matlab R2013a, Statistics package, The MathWorks Inc., Natick, MA.
} 
The family of metrics comprises all weightings of the three terms, reflecting uncertainty in the relative importance of each term to diversity in the morphological space. This family generalizes other similar metrics used on morphological spaces [41, 45].

While the algorithm implemented in Matlab allows entry of any prior on a 66 element equidistant discretization of $\Theta$, the OLDFAR program uses a uniform distribution as prior.

\section{Example output}

The following FAR output is based on a CGIAR, UNEP WCMC and FAO regional exploratory scenario study in South East Asia involving Cambodia, Laos and Vietnam, [76]. In a workshop process, as detailed in [76], regional stakeholders identified a large number of change factors and through voting and discussion selected the factors they considered to be most uncertain and most relevant for the scope of future agriculture and food security decision making.

Table 2: FAR factor and states example from a CGIAR South East Asia climate change, agriculture and food security regional scenarios workshop.

\begin{tabular}{|c|c|c|c|}
\hline Markets & Agriculture Investment & $\begin{array}{l}\text { Changing Land Use \& Soil } \\
\text { Quality }\end{array}$ & $\begin{array}{l}\text { Regulatory Enforcement } \\
\text { Capacity and Regional } \\
\text { Collaboration }\end{array}$ \\
\hline $\begin{array}{l}\text { M1: Laissez-faire: } \\
\text { unregulated market }\end{array}$ & $\begin{array}{l}\text { A1: Increasing/high public and } \\
\text { private }\end{array}$ & $\begin{array}{l}\text { L1: Climate Smart Agriculture } \\
\text { (CSA) practices with good soil } \\
\text { quality }\end{array}$ & $\begin{array}{l}\text { E1: Strong enforcement ability } \\
\text { with strong regional } \\
\text { collaboration }\end{array}$ \\
\hline $\begin{array}{l}\text { M2: Common regulated } \\
\text { market }\end{array}$ & $\begin{array}{l}\text { A2: Unbalanced; high private } \\
\text { and low public }\end{array}$ & $\begin{array}{l}\text { L2: Participatory/eco-friendly } \\
\text { land policy }\end{array}$ & $\begin{array}{l}\text { E2: Strong enforcement ability } \\
\text { with weak regional } \\
\text { collaboration }\end{array}$ \\
\hline \multirow[t]{2}{*}{$\begin{array}{l}\text { M3: Protectionism; } \\
\text { closed market }\end{array}$} & $\begin{array}{l}\text { A3: Decreasing/low public \& } \\
\text { private }\end{array}$ & $\begin{array}{l}\text { L3: Top-down/extant driven } \\
\text { land policy }\end{array}$ & $\begin{array}{l}\text { E3: Weak enforcement ability } \\
\text { with weak regional } \\
\text { collaboration }\end{array}$ \\
\hline & & $\begin{array}{l}\text { L4: Intensified crop yield with } \\
\text { poor soil quality. }\end{array}$ & \\
\hline
\end{tabular}

During the process the participants generate FAR "compatibility matrices" that indicate the pairwise compatibility of states between distinct factors. In this case there are 6 distinct pairs of the four factors, so participants generated 6 compatibility matrices. The descriptions in Table 2 , the choice of an initial scenario $(2,1,1,1)$, and the compatibility matrices are the inputs into the OLDFAR software interface. Based on the implementation as described in Section 4, OLDFAR generated the following output of multiple sets of six highly diverse scenarios.

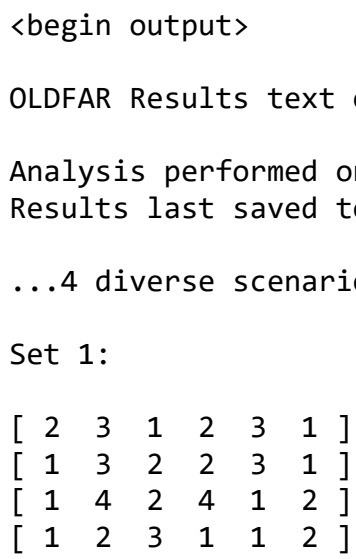


Initial Scenario... Markets:Common regulated market. Agricultural Investment:High public \& private. Changing Land Use \& Soil Quality:CSA practices with good soil quality. Enforcement Capacity \& Regional Collaboration:Strong enforcement ability with strong regional collaboration.

Scenario 2... Markets:Protectionism:closed market. Agricultural Investment:Low public \& private. Changing Land Use \& Soil Quality:Intisified crop yield with poor soil quality. Enforcement Capacity \& Regional Collaboration:Strong enforcement ability with low regional collaboration.

Scenario 3... Markets:Laissez-faire:unregulated. Agricultural Investment:Unbalanced: high private. Changing Land Use \& Soil Quality:Participatory/eco-friendly land policy. Enforcement Capacity \& Regional Collaboration:Weak enforcement ability with weak regional collaboration.

Scenario 4... Markets:Common regulated market. Agricultural Investment:Unbalanced: high private. Changing Land Use \& Soil Quality:Intisified crop yield with poor soil quality. Enforcement Capacity \& Regional Collaboration:Strong enforcement ability with strong regional collaboration.

Scenario 5... Markets:Protectionism:closed market. Agricultural Investment:Low public \& private. Changing Land Use \& Soil Quality:CSA practices with good soil quality. Enforcement Capacity \& Regional Collaboration:Strong enforcement ability with strong regional collaboration.

Scenario 6... Markets:Laissez-faire:unregulated. Agricultural Investment:High public \& private. Changing Land Use \& Soil Quality:Participatory/eco-friendly land policy. Enforcement Capacity \& Regional Collaboration:Strong enforcement ability with low regional collaboration.

Set 2:

$\left[\begin{array}{lllllll}2 & 3 & 1 & 2 & 3 & 1 & ] \\ {[} & 1 & 3 & 2 & 2 & 3 & 3\end{array}\right]$

Initial Scenario... Markets:Common regulated market. Agricultural Investment:High public \& private. Changing Land Use \& Soil Quality:CSA practices with good soil quality. Enforcement Capacity \& Regional Collaboration:Strong enforcement ability with strong regional collaboration.

Scenario 2... Markets:Protectionism:closed market. Agricultural Investment:Low public \& private. Changing Land Use \& Soil Quality:Intisified crop yield with poor soil quality. Enforcement Capacity \& Regional Collaboration:Strong enforcement ability with low regional collaboration.

Scenario 3... Markets:Laissez-faire:unregulated. Agricultural Investment:Unbalanced: high private. Changing Land Use \& Soil Quality:Participatory/eco-friendly land policy. Enforcement Capacity \& Regional Collaboration:Weak enforcement ability with weak regional collaboration.

Scenario 4... Markets:Common regulated market. Agricultural Investment:Unbalanced: high private. Changing Land Use \& Soil Quality:Intisified crop yield with poor soil quality. Enforcement Capacity \& Regional Collaboration:Strong enforcement ability with strong regional collaboration.

Scenario 5... Markets:Protectionism:closed market. Agricultural Investment:Low public \& private. Changing Land Use \& Soil Quality:CSA practices with good soil quality. Enforcement Capacity \& Regional Collaboration:Strong enforcement ability with strong regional collaboration.

Scenario 6... Markets:Laissez-faire:unregulated. Agricultural Investment:Low public \& private. Changing Land Use \& Soil Quality:Participatory/eco-friendly land policy. Enforcement Capacity \& Regional Collaboration:Strong enforcement ability with low regional collaboration.

Set 3:

$\left[\begin{array}{llllll}2 & 1 & 3 & 3 & 1 & 2\end{array}\right]$

$\left[\begin{array}{llllll}1 & 3 & 3 & 3 & 1 & 2\end{array}\right]$

$\left[\begin{array}{llllll}1 & 2 & 4 & 1 & 2 & 3\end{array}\right]$

$\left[\begin{array}{llllll}1 & 3 & 1 & 2 & 2 & 1\end{array}\right]$

Initial Scenario... Markets:Common regulated market. Agricultural Investment:High public \& private. Changing Land Use \& Soil Quality:CSA practices with good soil 
quality. Enforcement Capacity \& Regional Collaboration:Strong enforcement ability with strong regional collaboration.

Scenario 2... Markets:Laissez-faire: unregulated. Agricultural Investment:Low public \& private. Changing Land Use \& Soil Quality:Participatory/eco-friendly land policy. Enforcement Capacity \& Regional Collaboration:Weak enforcement ability with weak regional collaboration.

Scenario 3... Markets:Protectionism:closed market. Agricultural Investment:Low public \& private. Changing Land Use \& Soil Quality:Intisified crop yield with poor soil quality. Enforcement Capacity \& Regional Collaboration:Strong enforcement ability with strong regional collaboration.

Scenario 4... Markets:Protectionism:closed market. Agricultural Investment:Low public \& private. Changing Land Use \& Soil Quality:CSA practices with good soil quality. Enforcement Capacity \& Regional Collaboration:Strong enforcement ability with low regional collaboration.

Scenario 5... Markets:Laissez-faire:unregulated. Agricultural Investment:High public \& private. Changing Land Use \& Soil Quality:Participatory/eco-friendly land policy. Enforcement Capacity \& Regional Collaboration:Strong enforcement ability with low regional collaboration.

Scenario 6... Markets:Common regulated market. Agricultural Investment:Unbalanced: high private. Changing Land Use \& Soil Quality:Top-down/extant driven land policy. Enforcement Capacity \& Regional Collaboration:Strong enforcement ability with strong regional collaboration.

Set 4:

$\left[\begin{array}{llllll}2 & 1 & 3 & 3 & 1 & 2\end{array}\right]$

$\left[\begin{array}{llllll}1 & 3 & 3 & 3 & 1 & 2\end{array}\right]$

$\left[\begin{array}{llllll}1 & 2 & 4 & 1 & 2 & 4\end{array}\right]$

$\left[\begin{array}{llllll}1 & 3 & 2 & 1 & 2 & 1\end{array}\right]$

Initial Scenario... Markets:Common regulated market. Agricultural Investment:High public \& private. Changing Land Use \& Soil Quality:CSA practices with good soil quality. Enforcement Capacity \& Regional Collaboration:Strong enforcement ability with strong regional collaboration.

Scenario 2... Markets:Laissez-faire:unregulated. Agricultural Investment:Low public \& private. Changing Land Use \& Soil Quality:Participatory/eco-friendly land policy. Enforcement Capacity \& Regional Collaboration:Weak enforcement ability with weak regional collaboration.

Scenario 3... Markets:Protectionism:closed market. Agricultural Investment:Low public \& private. Changing Land Use \& Soil Quality: Intisified crop yield with poor soil quality. Enforcement Capacity \& Regional Collaboration:Strong enforcement ability with low regional collaboration.

Scenario 4... Markets:Protectionism:closed market. Agricultural Investment:Low public \& private. Changing Land Use \& Soil Quality:CSA practices with good soil quality. Enforcement Capacity \& Regional Collaboration:Strong enforcement ability with strong regional collaboration.

Scenario 5... Markets:Laissez-faire:unregulated. Agricultural Investment:High public \& private. Changing Land Use \& Soil Quality:Participatory/eco-friendly land policy. Enforcement Capacity \& Regional Collaboration:Strong enforcement ability with low regional collaboration.

Scenario 6... Markets:Common regulated market. Agricultural Investment:Unbalanced: high private. Changing Land Use \& Soil Quality: Intisified crop yield with poor soil quality. Enforcement Capacity \& Regional Collaboration:Strong enforcement ability with strong regional collaboration.

<end output>

The OLDFAR software also provides a graphical representation of the diversity scores of the sets, which provides insight into why the algorithm chose the sets. With a small set of feasible scenarios sets 1, 2 and 4 are similar with small perturbations among the scenarios. Participants examined the sets and, through discussion about the skeleton narratives of the scenarios displayed in the output, went forward with set 3 . Subsequent workshop processes altered the scenario set to the final set found in [76]. 
The following FAR output is based on a CGIAR, UNEP-WCMC and UCI regional exploratory scenario study in the Andes involving Bolivia, Columbia, Ecuador and Peru, [77]. In a similar workshop process to the South East Asia study, as detailed in [77], regional stakeholders identified change factors, states, and compatibility matrices.

Table 3: FAR factor and states example from a CGIAR Andes climate change, agriculture and food security regional scenarios workshop. (Translated from original workshop and output in Spanish).

\begin{tabular}{|l|l|l|l|}
\hline Political power of State & Markets & Consumption Patterns & $\begin{array}{l}\text { Economic development and } \\
\text { inequality }\end{array}$ \\
\hline P1: Centralized & M1: Sustainable and regulated & C1:Subsistence (low income) & $\begin{array}{l}\text { E1: high economic growth and } \\
\text { specialization }\end{array}$ \\
\hline P2: Decentralized & $\begin{array}{l}\text { M2: Sustainable and } \\
\text { unregulated }\end{array}$ & $\begin{array}{l}\text { C2:Need (high income, } \\
\text { sustainable consuming) }\end{array}$ & $\begin{array}{l}\text { E2: low development and } \\
\text { specialization }\end{array}$ \\
\hline & $\begin{array}{l}\text { M3: Not sustainable and } \\
\text { unregulated }\end{array}$ & $\begin{array}{l}\text { C3:Wealthy (high income, } \\
\text { consumer) }\end{array}$ & $\begin{array}{l}\text { E3: high economic growth and } \\
\text { diversification }\end{array}$ \\
\hline & $\begin{array}{l}\text { M4: Not sustainable and } \\
\text { regulated }\end{array}$ & $\begin{array}{l}\text { E4: low development and } \\
\text { diversification }\end{array}$ \\
\hline & &
\end{tabular}

OLDFAR generated the following output of multiple sets of six highly diverse scenarios.

<begin output>

OLDFAR Results text output

Analysis performed on: 27-Nov-2013 22:39:06. Data last saved to: OLDFARsavechange.mat.

Set 1:

$\left[\begin{array}{lllllll}2 & 1 & 1 & 2 & 2 & 1\end{array}\right]$

$\left[\begin{array}{llllll}3 & 1 & 4 & 2 & 4 & 2\end{array}\right]$

$\left[\begin{array}{llllll}2 & 3 & 1 & 3 & 1 & 3\end{array}\right]$

$\left[\begin{array}{llllll}2 & 4 & 1 & 3 & 4 & 1\end{array}\right]$

Initial Scenario ... Political power: Decentralized. Markets: Not sustainable and unregulated. Consumption Patterns: Subsistence (low income). Economic Development and inequality: low development and specialization.

Scenario 2 ... Political power: Centralized. Markets: Sustainable and regulated. Consumption Patterns: Need (high income, sustainable consuming). Economic Development and Inequality: low development and diversification.

Scenario 3 ... Political power: Centralized. Markets: Not sustainable and regulated. Consumption Patterns: Wealthy (high income, consumer). Economic Development and Inequality: high economic growth and specialization.

Scenario 4 ... Political power: Decentralized. Markets: Sustainable and unregulated. Consumption Patterns: Need (high income, sustainable consuming). Economic Development and Inequality: high economic growth and diversification.

Scenario 5 ... Political power: Decentralized. Markets: Not sustainable and regulated. Consumption Patterns: Wealthy (high income, consumer). Economic Development and Inequality: low development and diversification.

Scenario 6 ... Political power: Centralized. Markets: Sustainable and unregulated. Consumption Patterns: Need (high income, sustainable consuming). Economic Development and Inequality: high economic growth and specialization.

Set 2:

$\left[\begin{array}{lllllll}2 & 1 & 1 & 2 & 2 & 2 & ] \\ {[} & 3 & 1 & 4 & 2 & 4 & 3\end{array}\right]$


Initial Scenario ... Political power: Decentralized. Markets: Not sustainable and unregulated. Consumption Patterns: Subsistence (low income). Economic Development and Inequality: low development and specialization.

Scenario 2 ... Political power: Centralized. Markets: Sustainable and regulated. Consumption Patterns: Need (high income, sustainable consuming). Economic Development and Inequality: low development and diversification.

Scenario 3 ... Political power: Centralized. Markets: Not sustainable and regulated. Consumption Patterns: Wealthy (high income, consumer). Economic Development and Inequality: high economic growth and diversification.

Scenario 4 ... Political power: Decentralized. Markets: Sustainable and unregulated. Consumption Patterns: Need (high income, sustainable consuming). Economic Development and Inequality: high economic growth and specialization.

Scenario 5 ... Political power: Decentralized. Markets: Not sustainable and regulated. Consumption Patterns: Wealthy (high income, consumer). Economic Development and Inequality: high economic growth and specialization.

Scenario 6 ... Political power: Decentralized. Markets: Not sustainable and unregulated. Consumption Patterns: Wealthy (high income, consumer). Economic Development and Inequality: low development and diversification.

Set 3:

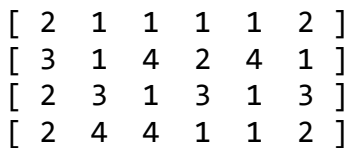

Initial Scenario ... Political power: Decentralized. Markets: Not sustainable and unregulated. Consumption Patterns: Subsistence (low income). Economic Development and Inequality: low development and specialization.

Scenario 2 ... Political power: Centralized. Markets: Sustainable and regulated. Consumption Patterns: Need (high income, sustainable consuming). Economic Development and Inequality: low development and diversification. Scenario 3 ... Political power: Centralized. Markets: Not sustainable and regulated. Consumption Patterns: Wealthy (high income, consumer). Economic Development and Inequality: low development and diversification.

Scenario 4 ... Political power: Centralized. Markets: Sustainable and unregulated. Consumption Patterns: Need (high income, sustainable consuming). Economic Development and Inequality: high economic growth and specialization.

Scenario 5 ... Political power: Centralized. Markets: Not sustainable and regulated. Consumption Patterns: Wealthy (high income, consumer). Economic Development and Inequality: high economic growth and specialization.

Scenario 6 ... Political power: Decentralized. Markets: Sustainable and regulated. Consumption Patterns: Need (high income,

sustainable consuming). Economic Development and Inequality: low development and specialization.

Set 4 :

$\left[\begin{array}{lllllll}2 & 1 & 1 & 2 & 2 & 1\end{array}\right]$

$\left[\begin{array}{llllll}3 & 1 & 4 & 2 & 4 & 3\end{array}\right]$

$\left[\begin{array}{llllll}2 & 3 & 1 & 3 & 1 & 2\end{array}\right]$

$\left[\begin{array}{llllll}2 & 4 & 1 & 1 & 4 & 4\end{array}\right]$

Initial Scenario ... Political power: Decentralized. Markets: Not sustainable and unregulated. Consumption Patterns: Subsistence (low income). Economic Development and Inequality: low development and specialization.

Scenario 2 ... Political power: Centralized. Markets: Sustainable and regulated. Consumption Patterns: Need (high income, sustainable consuming). Economic Development and Inequality: low development and diversification.

Scenario 3 ... Political power: Centralized. Markets: Not sustainable and regulated. Consumption Patterns: Wealthy (high income, consumer). Economic Development and Inequality: high economic growth and specialization.

Scenario 4 ... Political power: Decentralized. Markets: Sustainable and unregulated. Consumption Patterns: Need (high income, sustainable consuming). Economic Development and Inequality: high economic growth and specialization.

Scenario 5 ... Political power: Decentralized. Markets: Not sustainable and regulated. Consumption Patterns: Wealthy (high income, consumer). Economic Development and Inequality: low development and diversification. 
Scenario 6 ... Political power: Centralized. Markets: Not sustainable and unregulated. Consumption Patterns: Subsistence (low income). Economic Development and Inequality: low development and diversification.

With 44 feasible scenarios, the diverse sets of 6 scenarios are more diverse between themselves than the first example. Participants examined the sets and, through discussion about the skeleton narratives of the scenarios displayed in the output, went forward with set 1 . Subsequent workshop processes altered the scenario set to the final set found in [77]. The interested reader can also examine the final policy initiatives and discussions stimulated by the scenarios sets 4 .

\section{Results and discussion}

The mathematics in the OLDFAR technique is only for leverage on the number of combinations of choices from a large set of plausible scenarios. Human judgment makes the final decision on what is the most useful set of scenarios to go forward with for exploratory analysis.

For the CCAFS regional scenario program this leverage has proven useful even when the number of combinations is not as large as the CCAFS South Asia study. In South East Asia, the Andes and Central America, only four factors were used, allowing for 3-6 states per factor instead. This still allowed for a large possibility space for which the OLDFAR tool was needed to help select maximally diverse scenario sets.

Further results on the use of OLDFAR in the CCAFS scenarios program will be available in a separate publication. Some key lessons learned through the application of the OLDFAR method in four regional case studies are:

1. The need for factors and states to be defined clearly and agreed upon by all involved in the scenario development is important. If this is not done it makes the compatibility analysis unclear and contentious.

2. We found that for exploratory scenario studies states that fall between two extremes should be avoided (that is, through practice we ended up avoiding states such as those shown in the first five factors in Table 1), Their perceived compatibility with other states is usually higher, and this leads to an overrepresentation of "middle of the road" scenarios that stick closely to participants' bias or "phantom scenario", not leading to sufficiently distinct and diverse scenarios. CCAFS scenarios have been used to question the assumptions of policies and plans in a given region. This makes a set of more extreme but still plausible scenarios more useful, because they are more likely to lead to an examination of the assumptions made in a policy or plan, and where it might fall short, than 'middle of the road' scenarios - and more likely to offer complementary insights as a set. Where possible, most factor states should be qualitatively different from each other, rather than representing a continuum. Uncertainty whether factors states for specific studies are qualitatively different, or represent a continuum, is one of the features captured by the family of metrics in Section 4.1.

\footnotetext{
${ }^{4}$ CCAFS blog, https://ccafs.cgiar.org/blog/andean-countries-join-forces-strengthen-policies-face-futurescenarios\#.Vchf8_mqqko
} 
3. Practical considerations about the time available to review factor state interactions, and the ease with which a scenario set is communicated proved important. In the first process where an early version OLDFAR was used in South Asia, six factors with two states each were used. This resulted in a useful set of scenarios, but one that sometimes proved fairly difficult to communicate to new audiences because they had to grasp the interaction of six factors for each of the scenarios. It also did not allow the process to benefit from the possibility of having more than two states per factor. Therefore, in later processes in Central America and the examples given here for Southeast Asia and the Andes region, the choice was made to focus on fewer factors, but emphasize the possibility for a larger number of states. This way, the process emphasized that each factor could develop in a number of directions depending on how it was framed, and participants found the final scenario set more intelligible.

4. Even though OLDFAR produces sets of scenarios constructed only out of factor state combinations that process participants found plausible or were at least willing to consider, the combination of a string of states may lead participants to question its plausibility as a complete structure for a given scenario. Process participants should be given space to question and critically review OLDFAR outputs to ensure that they consider the combinations of factor states that are to be taken forward into scenarios plausible.

5. Since this tool has been designed from a plausibility perspective, it is important to note that plausibility depends on how scenario narratives describe the interactions and developments of factors. A combination of factors that might at first glance be considered implausible by scenario developers and users may be rendered plausible through its narrative. This is important, since one of the major uses of scenarios lies in broadening decision-makers' perspectives on the future and moving them beyond existing biases. OLDFAR aids this by allowing scenarios with very low plausibility scores to be included in exploratory scenario sets.

6. Conceptual complexity in the meaning and description of factors and states in multidimensional morphological analysis can make heuristic examination of exploratory scenarios difficult even in small plausible scenarios sets. Previously we have encountered a FAR analysis with four dimensions but only 22 plausible scenarios. After a heuristic assessment analysts were adamant that a set of 3 scenarios extracted from the 22 represented the key diverse trajectories for the exploratory analysis.

Mathematical analysis however found a fourth scenario, containing a combination of states missed in the heuristic assessment, which was sufficiently interesting, plausible and diverse from the existing three that it was admitted to be explored.

\section{Conclusion}

The OLDFAR technique is a method to extract exploratory scenarios from a multi-dimensional morphological space. It takes into account uncertainty in the participant's perception of diversity between the exploratory scenarios and the qualitative nature of scenario narratives. It outputs a $n$ set of $k$ plausable future scenarios. Each set of $k$ plausable future scenarios robustly minimizes (robust to uncertainty as programmed by a family of metrics) the futures not being explored when extracting $k$ scenarios from the initial morphological space. The $n$ choices represent distinct combined narratives. Human judgment or a subsequent analytical method 
can assess the diversity and combined narratives and decide which single set of $k$ plausable future scenarios to proceed with as exploratory scenarios.

Through its use in four regional case studies, OLDFAR has generated much enthusiasm among those who participated in processes where the method was applied - with many in governments, NGOs and research organizations showing interest in using the method for themselves. In addition, scenarios researchers are recognizing the limits of two-axes scenario development and are exploring more multi-dimensional scenario development approaches. The theory and application of OLDFAR can contribute to the state of the art of scenario development.

Future technical work involves adding an a posterior preference choosing interface, and improvements to the OLDFAR algorithm. User input through the OLDFAR program would enable interactive optimization methods with adaptive boosting [75]. A future OLDFAR program could benefit from computational efficiencies and an evaluation of alternative optimization methods.

Future research into the application of OLDFAR could include a greater understanding of how different FAR scores affect the usefulness of the scenarios; research into the analytic processes participants go through in the different steps involved; the trade-offs between more or less factors and more or less states for the scenarios' usefulness, and the challenges and opportunities in communicating multi-factor, multi-state scenarios to new users. OLDFAR aids exploratory scenario methods where the emphasis is on diversity and likely or unlikely is irrelevant. We are researching the use of secondary probability methods to capture both plausibility and probability from participants in scenario studies.

\section{Acknowledgements}

This research was supported by the CGIAR CCAFS research program, the FAO EPIC program and UNEP WCMC.

\section{References:}

[1] S.J. Vermeulen, et al., Addressing uncertainty in adaptation planning for agriculture, Proceedings of the National Academy of Sciences of the United States of America, 110 (2013) 8357-8362.

[2] J.M. Vervoort, et al. ,Exploring dimensions, scales, and cross-scale dynamics from the perspective of change agents in social-ecological systems, Ecology and Society, 17 (2012).

[3] M. Gibbons, Science's new social contract with society, Nature, 402 (1999) C81-C84.

[4] Vervoort, J.M., et al., Challenges to scenario-guided adaptive action on food security under climate change, Global Environmental Change, (2014).

[5] K. Kok, M. van Vliet, I. Bärlund, A. Dubel, J. Sendzimir, Combining participative backcasting and exploratory scenario development: Experiences from the SCENES project, Technological Forecasting and Social Change, 78 (2011) 835-851.

[6] P.J.H. Schoemaker, Multiple scenario development: Its conceptual and behavioral foundation, Srateg. Manage. J., 14 (1993) 193-213.

[7] R.J. Lempert, D.J. Groves, S.W. Popper, S.C. Bankes, A general, analytic method for generating robust strategies and narrative scenarios, Management Science, 52 (2006) 514-528. 
[8] R. Bradfield, G. Wright, G. Burt, G. Cairns, K. van der Heijden, The origins and evolution of scenario techniques in long range business planning, Futures, 37 (2005) 795-812.

[9] M. Godet, The Art of Scenarios and Strategic Planning: Tools and Pitfalls, Technologcial Forecasting \& Social Change, 65 (2000) 3-22.

[10] P.P. Schwartz, The Art of the Long View: Planning for the Future in an Uncertain World, Currency Doubleday, New York, NY, 1996.

[11] F. Zwicky, Discovery, Invention, Research, through the Morphological Approach, Macmillan, New York, 1969.

[12] M. Godet, Creating Futures: Scenario Planning as a Strategic Management Tool, in, Economica, Paris, 2006.

[13] T. Ritchey, Problem Structuring using Computer-Aided Morphological Analysis, Journal of the Operational Research Society, 57 (2006) 792-801.

[14] R. Rhyne, Field anomaly relaxation: The arts of usage, Futures, 27 (1995) 657-674.

[15] R. Rhyne, Whole-pattern futures projection, using field anomaly relaxation, Technological Forecasting and Social Change, 19 (1981) 331-360.

[16] R.G. Coyle, R. Crawshay, L. Sutton, Futures assessment by field anomaly relaxation: A review and appraisal, Futures, 26 (1994) 25-43.

[17] S.A. van't Klooster, M.B.A. van Asselt, Practicing the scenario-axis technique, Futures, 38 (2006) 15-30.

[18] K. van der Heijden, Scenarios: The art of strategic conversation, John Wiley \& Sons, Chichester, UK, 2005.

[19] A. Wilkinson, E. Eidinow, Evolving practices in environmental scenarios: a new scenario typology, Environmental Research Letters, 3 (2008) 045017.

[20] A. Wilkinson, R. Kupers, The essence of scenarios: learning from the Shell experience, Amsterdam University Press, Amsterdam, 2014.

[21] P.W.F. van Notten, A.M. Sleegers, M.B.A. van Asselt, The future shocks: On discontinuity and scenario development, Technological forecasting and social change, 72 (2005) 175-194.

[22] R. Ramírez, C. Selin, Plausibility and probability in scenario planning, Foresight, 16 (2014) 54-74.

[23] G.A. Miller, The magical number seven, plus or minus two: Some limits on our capacity for processing information, Psychological Review, 63 (1956) 81-97.

[24] I. Kaplansky, Set theory and metric spaces, Allyn and Bacon, Boston, 1972.

[25] P.M. Lee, Bayesian statistics: an introduction, Oxford University Press, New York, 1989.

[26] W.M. Bolstad, Introduction to Bayesian statistics, John Wiley \& Sons, Hoboken, 2007.

[27] E.T. Jaynes, Information theory and statistical mechanics, Physical Review. Series II, 106 (1957) 620-630.

[28] S. Guiasu, A. Shenitzer, The principle of maximum entropy, The Mathematical Intelligencer, 7 (1985) 42-48.

[29] E.T. Jaynes, G.L. Bretthorst, Probability theory: the logic of science, Cambridge University Press, Cambridge, 2003.

[30] K. Miettinen, Nonlinear Multiobjective Optimization, Springer, Boston, 1998.

[31] R.T. Marler, J.S. Arora, Survey of multi-objective optimization methods for engineering, Struct Multidisc Optim, (2004) 369-395.

[32] M. Ehrgott, Multicriteria Optimization, Springer, Berlin, 2005.

[33] R.J. Lempert, M.T. Collins, Managin the risk of uncertain threshold response: comparison of robust, optimum, and precautionary approaches, Risk Analysis, 27 (2007) 1009-1026.

[34] A. Ben-Tal, A. Nemirovski, Robust solutions of linear programming problems contaminated with uncertain data, Mathematical Programming, 88 (2008) 411-424.

[35] A.S. Dymond, S. Kok, P.S. Heyns, The sensitivity of multi-objective optimization algorithm performance to objective function evaluation budget, in: 2013 IEEE Congress on Evolutionary Computation, Cancun, 2013, pp. 1868-1875.

[36] L.A. Cox, Confronting Deep Uncertainties in Risk Analysis, Risk Analysis, 32 (2012) 16071629. 
[37] C. Hamarat, J.H. Kwakkel, E. Pruyt, Adaptive Robust Design under deep uncertainty, Technological Forecasting \& Social Change, 80 (2013) 408-418.

[38] W.E. Walker, V.A.W.J. Marachu, D. Swanson, Addressing deep uncertainty using adaptive policies: Introduction to section 2, Technological Forecasting \& Social Change, 77 (2010) 917923.

[39] M. Haasnoot, J.H. Kwakkel, W.E. Walker, J.t. Maat, Dynamic adaptive policy pathways: A method for crafting robust decisions for a deeply uncertain world, Global Environmental Change, 23 (2013) 485-498.

[40] R.J. Lempert, D.G. Groves, Identifying and evaluating robust adaptive policy responses to climate change for water management agencies in the American West, Technological Forecasting and Social Change, 77 (2010) 960-974.

[41] 0. Tietje, Identification of a small reliable and efficient set of consistent scenarios, Eur. J. Oper. Res., 162 (2005) 418-432.

[42] B.P. Bryant, R.J. Lempert, Thinking inside the box: A participatory, computer-assisted approach to scenario discovery, Technological Forecasting \& Social Change, 77 (2010) 34-49. [43] J.H. Kwakkel, W.L. Auping, E. Pruyt, Dynamic scenario discovery under deep uncertainty: The future of copper, Technological Forecasting \& Social Change, 80 (2013) 789-800.

[44] M. Godet, Methods of prospective $>$ Softwares $>$ Smic-Prob Expert : La prospective, in, axninformatique, 2010.

[45] H. Carlsen, E.A. Eriksson, K.H. Dreborg, B. Johansson, O. Bodin, Scenario Diversity Analysis for systematic exploration of scenario spaces, (2014).

[46] L.A. Zadeh, Fuzzy Sets, Information and Control, 8 (1965) 338-353.

[47] D. Dubois, H. Prade, Fuzzy sets and systems, Academic Press, New York, 1988.

[48] A. Shen, N.K. Vereshchagin, Basic set theory, American Mathematical Society, Providence, 2002.

[49] A.W. Knapp, Basic Real Analysis, Birkhauser, Boston, 2005.

[50] L. Cohen, An introduction to the philosophy of induction and probability, Oxford University Press, New York, 1989.

[51] H. Bauer, Probability Theory, De Gruyter, Berlin, 1996.

[52] R.M. Dudley, Real Analysis and Probability, Cambridge University Press, Cambridge, 2002.

[53] J.C. Taylor, An introduction to measure and probability, Springer, New York, 1997.

[54] V.I. Bogachev, Measure theory, Springer, New York, 2007.

[55] C.E. Shannon, A mathematical theory of communication, Bell System Technical Journal, 27 (1948) 379-423 \& 623-656.

[56] R.F. Hoskins, Delta functions: an introduction to generalised functions, Oxford University Press, Woodhead, 2011.

[57] F.G. Friedlander, Introduction to the theory of distributions, Cambridge University Press, Cambridge, 1982.

[58] Y. Jin, B. Sendhoff, Trade-off between performance and robustness: an evolutionary multiobjective approach, in, Evolutionary Multi-Criteria Optimization, Springer, Berlin Heidelberg, 2003, pp. 237-251.

[59] J.E. Fieldsend, R.M. Everson, Multi-objective optimisation in the presence of uncertainty, in: Evolutionary Computation, 2005. The 2005 IEEE Congress on, Edinburgh, Scotland, 2005, pp. 243-250.

[60] Y. Jin, J. Branke, Evolutionary optimization in uncertain environments-a survey, Evolutionary Computation, IEEE Transactions on, 9 (2005) 303-317.

[61] K. Deb, H. Gupta, Introducing Robustness in Multi-Objective Optimization, Evolutionary Computation, 14 (2006) 463-494.

[62] H.M. Markowitz, G.P. Todd, Mean-variance analysis in portfolio choice and captial markets, Wiley, Hoboken, 2000.

[63] A. Ben-Tal, L. El Ghaoui, A. Nemirovski, Robust Optimization, Princeton University Press, Princeton, 2009.

[64] D. Bertsimas, D.B. Brown, C. Caramanis, Theory and applications of robust optimization, SIAM Review, 53 (2011) 464-501. 
[65] G. Loomes, R. Sugden, Regret theory: An alternative theory of rational choice under uncertainty, Economic Journal, 92 (1982) 805-824.

[66] A.M. Geffrion, A parametric programming solution to the vector maximum problem, with applications to decision under uncertainty, in, Stanford Univ Graduate School of Business, Stanford, 1965.

[67] L.J. Savage, The foundations of statistics, John Wiley \& Sons, New York, 1954.

[68] J. Figueira, S. Greco, M. Erghott, Multiple criteria decision analysis, Springer, New York, 2005.

[69] M. Shaked, J.G. Shanthikumar, Stochastic orders and their applications, Academic Press, Boston, 1994.

[70] A.W. Marshall, Multivariate stochastic orderings and generating cones of functions, in: K.C. Mosler, M. Scarsini (Eds.), Stochastic orders and decision under risk, Institute of Mathematical Statistics, Hayward, 1991, pp. 231-247.

[71] P.J.M. van Laarhoven, E.H.L. Aarts, Simulated annealing : theory and applications, Kluwer Academic Publishers, Norwell, MA, U.S.A., 1987.

[72] K. Meittinen, Introduction to Multiobjective Optimization: Noninteractive Approaches, in: J. Branke, K. Deb, K. Miettinen, R. Slowinski (Eds.), Multiobjective Optimization, Springer, Berlin Heidelberg, 2008, pp. 1-26.

[73] B. Roy, V. Mousseau, A theoretical framework for analysing the notion of relative importance of criteria, Journal of Multi-Criteria Decision Analysis, 5 (1996) 145-159.

[74] J. Figueira, B. Roy, Determining the weights of criteria in the ELECTRE type method with a revised Simos' procedure, European Journal of Operational Research, 139 (2002) 317-326. [75] S. Minghe, A. Stam, R. Steuer, E., Interactive multiple objective programming using Tchebycheff programs and artifical neural networks, Computers \& Operations Research, 27 (2000) 601-620.

[76] D. Mason-D'Croz, et al., Multi-factor, multi-state, multi-model scenarios: exploring food and climate futures for Southeast Asia, submitted to Environmental Modelling and Software, 2015

[77] CCAFS, Taller para la construcción de

Escenarios Socioeconomicos, Cali, Colombia, 27-29 Novembre 2013, CCAFS Report (in Spanish), https://cgspace.cgiar.org/rest/bitstreams/31970/retrieve 


\section{Technical Appendix}

\subsection{OLDFAR Software Algorithms}

Implementing the OLDFAR method for the three-criterion $E_{w}, E_{b}, 1-V$-optimization requires determining the diversity score (the distribution on the square) of every $n$ set of $k$ scenarios $P S_{n, k}$, removing dominated sets, finding the Pareto front in the cube, and then executing a preferential choice from the Pareto front. Doing this directly is infeasible for many morphological analyses. For the software implementation we used the following algorithm:

1) INPUT: FAR

2) INPUT: Initial Scenario

3) Collect maximal diversity sets of $k$ scenarios for each individual metric.

4) Perform Robust Optimization (2-criteria) on within diversity in this collection of $k$ scenario sets. Collect the Pareto front and sets near the Pareto front.

5) Calculate total diversity score of $n$ sets from collection of $k$ scenarios sets.

6) Perform Robust Optimization (3-criteria) on total diversity score of collections.

7) Pick one $n$ set from Pareto front. Preference used to pick from front is controlled by user in analysis options.

8) OUTPUT: Human judgment picks one of the sets of $k$ scenarios from within the $n$ set based on narrative.

9) OUTPUT: Output is a set of $k$ plausible scenarios for exploratory analysis.

Step 3 uses a stepwise algorithm, starting with the initial scenario $s_{0}$, to find maximal diversity subsets of $k$ scenarios for a fixed $\theta \in \Theta$. That is, for each $s \in \operatorname{argmax} \rho\left(\theta, \cdot,\left\{s_{0}\right\}\right)$ create the two sets $\left\{s_{0}, s\right\}$ and iterate this procedure with the two sets $\left\{s_{0}, s\right\}$ in the place of $\left\{s_{0}\right\}$ until a collection $A_{\theta}$ of $k$ sets are created. Finding $\operatorname{argmax} d(\theta, \cdot)$ within the finite set $A_{\theta}$ would be the end of the OLDFAR method if there was no consideration of uncertainty in $\theta$. Because of the uncertainty, and that robustly maximal sets are not necessarily maximal sets for any fixed choice of $\theta$, we keep the entire collection $A_{\theta}$. The set $B=\cup_{\theta \in \Theta} A_{\theta}$ is a useful collection of initial candidates for robustly maximal sets since the sets it contains are very high or maximally scored for individual $\theta$ with a reduced chance of stochastically dominating each other.

Step 4 uses simulated annealing [71] to broaden the stock $B$ of robustly maximal candidates. It improves Pareto optimality according to maximizing $E$ and $1-V$ (with removal according to a weakened dominance as discussed). In OLDFAR the objective functions are complicated, statistics of distributions that have to be estimated for each generation. For this reason a 2criteria optimization is used to generate candidates for the 3-criteria optimization, rather than starting on the 3-criteria optimization directly.

Step 5 calculates the within and between diversity distribution of $n$ sets formed from the results of Step 4 and removes all dominated sets. We denote the remaining collection of $n$ sets of $k$ scenarios by $D$. Step 6 uses $D \subset P S_{n, k}$ as initial candidates for a simulated annealing approximation to the Pareto front in $P S_{n, k}$ for the three-criterion $E_{w}, E_{b}, 1-V$ optimization. Step 7 uses a preferential choosing algorithm to pick the $n$ set of $k$ scenarios from the Pareto front most representative of the preference. The preferential choosing algorithm implemented in OLDFAR is, to our knowledge, new, so we report it. 


\subsection{Soft Linear Preferencing}

The OLDFAR software implements an a priori preferential method requiring non-interactive approximation of the entire Pareto front $[31,72]$. The OLDFAR program gives users three alternative a priori preferences and uses the following mechanism to make the preferential choice.

Let $W:=\{w, b, r \geq 0: w+b+r=1\}$. A preference is a function $P: W \rightarrow[0,1]$ stating the emphasis or preference for the criteria of within diversity (w), between diversity (b), and robustness ( $\mathrm{r}$ ) as measured by the objective functions $E_{w}, E_{b}$, and $1-V$ respectively. We assume the Pareto front $\partial_{+}$to choose from has been normalized in the cube. Linear preferencing (or scalarization) assigns the preference $P(\bar{w}), \bar{w} \in W$, to the element of the Pareto front that maximizes the linear objective function $\bar{w} \cdot\left(E_{w}, E_{b}, 1-V\right)$ and chooses the element of the Pareto front that maximizes preference. In basic versions of scalarization the preference $P$ is supported on one weighting, or several candidate weightings [72]. How to choose the weightings, essentially reducing a multi-criteria trade-off problem to a linear single-criteria problem, is known to be problematic $[73,74]$. Useful alternatives take into account vague or approximate specification of human preference between criteria in trade-off and select robust preference maximizers from the Pareto front.

Soft linear preferencing (SLP) treats the preference $P$ as a fuzzy set of weightings and assigns preference to an element of the Pareto front in proportion to normalized shortest distance to maximal planes (those points that maximize a linear objective function lie in the maximal plane, so are accorded the highest preference). The element of the Pareto front chosen is that which maximizes the "belonging" to the preference. SLP uses a surface transformation $A_{S}: W \rightarrow$ $[0,1]^{3}, s \geq 1$, to adjust the spacing of the maximal planes. SLP uses a mapping $B_{t}: \partial_{+} \rightarrow$ $[0,1]^{3}, t \geq 1$, of the Pareto front to adjust linearity of the objective functions and to allow preferential choice of non-extremal points [30,72]. Mathematically, for each $\bar{w} \in W$ define the maximal planes and inverted distances to the maximal planes

$$
\begin{gathered}
M S_{s, t}(\bar{w}):=\left\{\bar{x} \in \mathbb{R}^{3}: A_{s}(\bar{w}) \cdot \bar{x}=\max _{\mathcal{A} \in \partial_{+}} A_{s}(\bar{w}) \cdot B_{t}\left(E_{w}(\mathcal{A}), E_{b}(\mathcal{A}),(1-V)(\mathcal{A})\right)\right\} \\
M_{s, t}(\mathcal{A}, \bar{w}):=\left(1+\min _{\bar{x} \in M S_{s, t}(\bar{w})}\left\|\bar{x}-B_{t}\left(E_{w}(\mathcal{A}), E_{b}(\mathcal{A}),(1-V)(\mathcal{A})\right)\right\|^{2}\right)^{-\frac{1}{2}}, \quad \mathcal{A} \in \partial_{+} .
\end{gathered}
$$

The preferential choice from the Pareto front is obtained from maximizing the integral

$$
\underset{\mathcal{A} \in \partial_{+}}{\operatorname{argmax}} \int_{W} P(\bar{w}) M_{s, t}(\mathcal{A}, \bar{w}) d \bar{w}
$$

Values for the parameters $s, t$ are ideally machine learned from a trial of human decision makers on test fronts. For the OLDFAR program we used,

$$
\begin{gathered}
A_{s}(w, b, r):=\left(w^{\frac{1}{s}}, b^{\frac{1}{s}}, r^{\frac{1}{s}}\right), \quad s=3,(w, b, r) \in W \\
B_{t}(x, y, z):=\left(x^{\frac{1}{t}}, y^{\frac{1}{t}}, z^{\frac{1}{t}}\right), \quad t=1.5,(x, y, z) \in[0,1]^{3}
\end{gathered}
$$


and the preferences specified on a discretization of $W$ in Figure 2. Power transformation of objective functions to allow preferential choice of non-extremal points is standard [31].

INSERT FIGURE 2 
Figures and Captions:

FIGURE 1

(a)

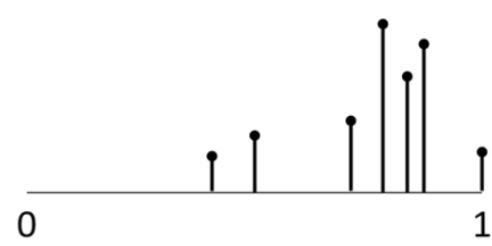

(c)

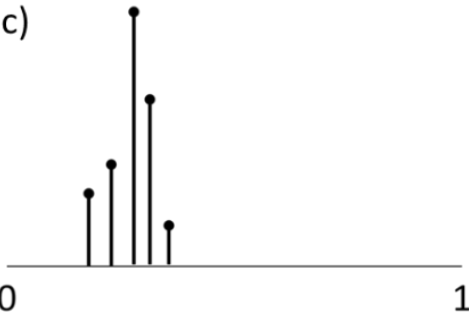

(b)

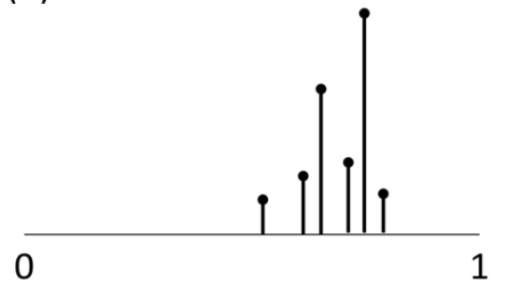

(d)

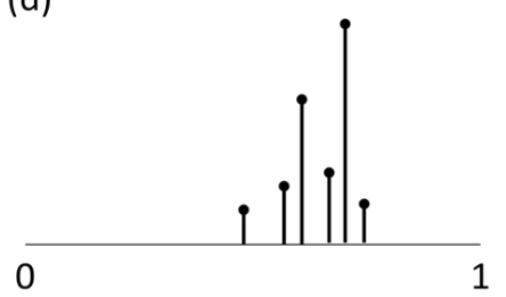

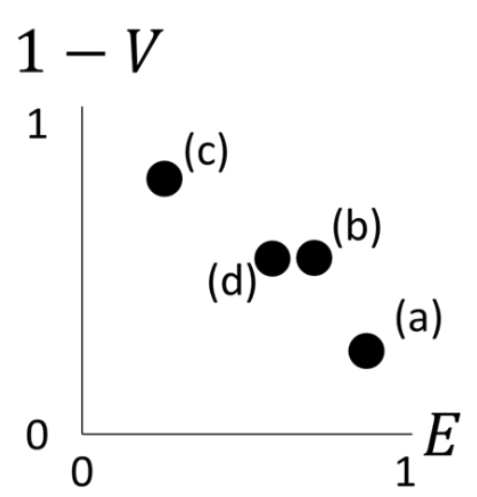

\section{FIGURE 1 CAPTION}

Figure 1: Examples of discrete distributions on $[0,1]$ and a plot of their expected value and one minus their variance. (a), (b) and (c) belong to the Pareto front. However, before the Pareto front should be considered a representation of robust high values (c) should be removed from consideration. It is stochastically dominated by at least one of the other distributions. (a) and (b) are in trade-off between high values and spread of values due to uncertainty. (d) is not on the Pareto front and, more strongly, is dominated by (b). However, it still has relatively robust high scores, and as a "near" robust optimal solution should be kept for assessment of the qualitative criteria of narrative. 
FIGURE 2

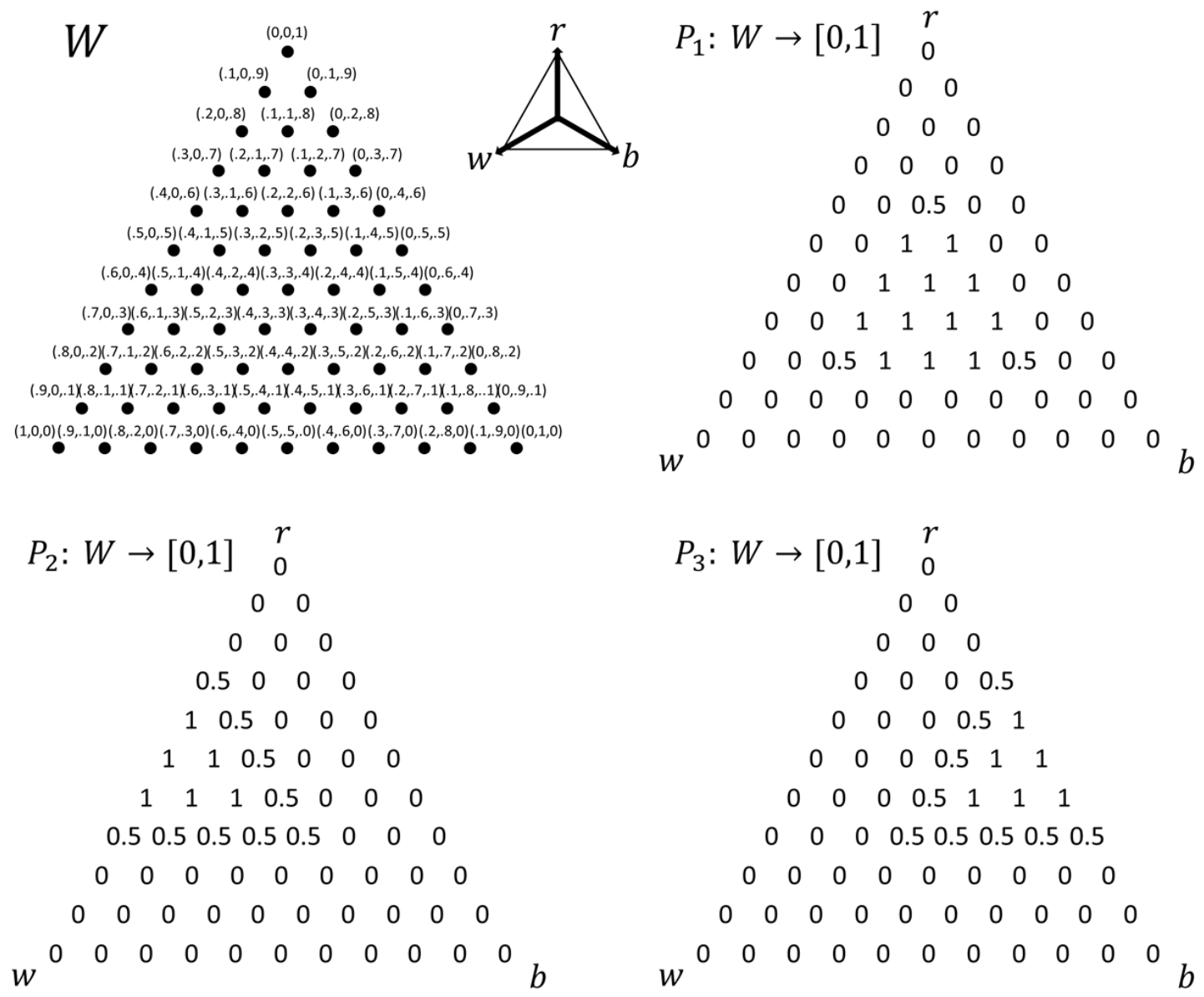

FIGURE 2 CAPTION

Figure 2: Top left panel is a 66 point equidistant discretization of the plane $W$ of weightings of within diversity $(w)$, between diversity $(b)$ and robustness to uncertainty $(r)$. Preferences for the three criteria can be depicted as triangles of values. $P_{1}$ preferences Pareto optimal solutions with robust within and between diversity, $P_{2}$ preferences more a robust high within diversity, and $P_{3}$ preferences more a robust high between diversity. 